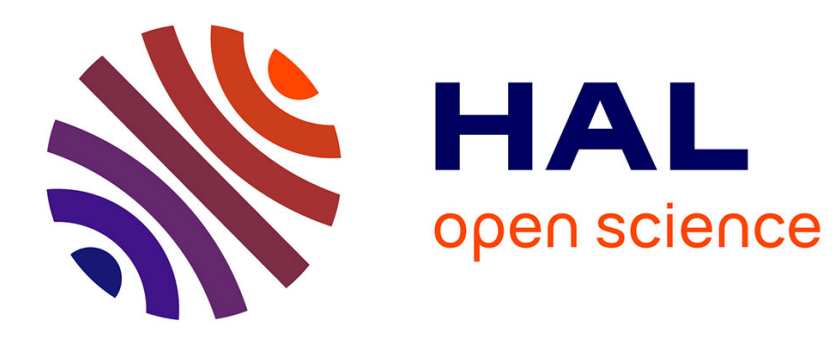

\title{
Les névroses mixtes: une leçon de méthode nosographique
}

François Villa

\section{To cite this version:}

François Villa. Les névroses mixtes : une leçon de méthode nosographique . L'Évolution Psychiatrique, 2007, L'évolution psychiatrique, 72 (2007) pp.477-487. 10.1016/j.evopsy.2007.07.004 . hal-01494503

\section{HAL Id: hal-01494503 \\ https://hal.science/hal-01494503}

Submitted on 24 Mar 2017

HAL is a multi-disciplinary open access archive for the deposit and dissemination of scientific research documents, whether they are published or not. The documents may come from teaching and research institutions in France or abroad, or from public or private research centers.
L'archive ouverte pluridisciplinaire HAL, est destinée au dépôt et à la diffusion de documents scientifiques de niveau recherche, publiés ou non, émanant des établissements d'enseignement et de recherche français ou étrangers, des laboratoires publics ou privés. 


\title{
Psychopathologie \\ Les névroses mixtes : une leçon de méthode nosographique
}

\section{Mixed neuroses: a lesson in methodical nosography}

\author{
François Villa* \\ Psychanalyste, Maître de Conférences en Sciences Humaines Cliniques, Université de Paris-VII-Denis-Diderot, \\ 26, rue de Paradis, 75480 Paris cedex 10, France \\ 30, boulevard de Strasbourg, 75010 Paris, France
}

Reçu le 13 novembre 2006 ; accepté le 13 juillet 2007

Disponible sur internet le 15 août 2007

\section{Résumé}

C'est en 1894 que Freud mentionne la notion de névrose mixte. L'auteur propose de revenir sur cette notion largement tombée en désuétude qui constitue une leçon de méthode clinique. Elle est à la croisée des chemins où Freud, en dégageant la méthode psychanalytique, se sépare tant de l'école allemande d'anatomopathologie (Meynert) que de l'école française de neuropathologie (Charcot) en accroissant la capacité diagnostique du clinicien. La reconnaissance de la dimension leurrante du tableau de la névrose mixte en permet la déconstruction et la construction par inférence de différents mécanismes psychiques. Ces derniers permettront la distinction de différentes affections psychiques. La décomposition du tableau de la névrose mixte introduit la dimension de la temporalité dans la constitution d'un ensemble symptomatique et rend envisageable une généalogie des formes névrotiques. Le symptôme n'est plus un objet dont l'évidence serait indiscutable du point de vue d'une perception objective. Il cesse d'être un trait distinctif par lui-même pour devenir un élément inscrit topiquement dans une dynamique où il est associé à d'autres éléments avec lesquels il constitue un complexe. Ce point critique est le lieu dynamique d'une activité pulsionnelle et le résultat d'une modification autoplastique, mais il est aussi une activité exercée sur certains objets du monde. Dans le traitement psychanalytique, penser et saisir le symptôme impose de découvrir sa fonction en devinant l'activité autoplastique et la modification alloplastique qu'il accomplit dans le transfert.

(C) 2007 Elsevier Masson SAS. Tous droits réservés.

\footnotetext{
* Auteur correspondant. (F. Villa).

Adresse e-mail : villa@univ-paris-diderot.fr (F. Villa).
} 


\begin{abstract}
Freud mentions the term of mixed neurosis in 1984. F. Villa goes back into this notion which has become obsolete and which is, in fact a lesson in clinical method. Freud, standing at crossroads, takes leave from the german school of anatomic-pathology (Meynert) and the french school of neuropathology by expanding the diagnostic capacity of the clinician. In doing so, he emphasizes the analytical method. To recognizie the illusion of this mixed neurosis makes possible deconstruction and reconstruction by gathering different psychic mechanisms. To deconstruct the setting of mixed neurosis brings in temporality in the bulding of a symptomatic whole and makes possible a genealogy of neurotic forms. The symptom is not anymore a fixed object, which would be inquestionable from the point of view of objective perception. It ceases to be a dinstinctive point in order to become a topographical component among a complex. This critical point is the dynamic scene of the instictual activity and the result of an autoplastic modification. It consists too in an action practise on some objects in the world. In analytical treatment, to think and to seize the symptom reveals its fonction by guessing the autoplastic activity and the alloplastic modification accomplished through transference.
\end{abstract}

(C) 2007 Elsevier Masson SAS. Tous droits réservés.

Mots clés : Diagnostic ; Mécanisme psychique ; Névroses actuelles ; Névrose mixte ; Nosographie ; Psychanalytique (mode de penser) ; Psychonévrose ; Symptôme ; Traitement psychique

Keywords: Diagnosis; Psychic mechanism; Actual neurosis; Mixed neurosis; Nosography; Neuropsychosis; Symptom; Psychic treatment

Nous voudrions revenir sur la notion largement tombée en désuétude de névrose mixte ${ }^{1}$. Il nous semble que l'on méconnaît trop souvent qu'elle est la croisée des chemins, où Freud, en dégageant la méthode psychanalytique, se sépare tant de l'école allemande d'anatomopathologie (Meynert) que de l'école française de neuropathologie (Charcot). La reconnaissance de la dimension leurrante du tableau symptomatologique manifeste que constitue la névrose mixte va en permettre la déconstruction et la construction par inférence de différents mécanismes psychiques. C'est à partir de ces derniers que différentes affections psychiques pourront être distinguées entre elles, chacune étant déterminée de manière caractéristique par la prédominance de l'un de ces mécanismes. C'est en 1894, dans "Les névropsychoses de défense » que Freud fait appel à la notion de névrose mixte pour indiquer pourquoi il est si difficile de distinguer les trois modes d'entrée dans la maladie qu'il a décrits :

«La survenue simultanée de phobies et de symptômes hystériques, qui est in praxis si fréquemment observée, appartient bel et bien aux facteurs qui rendent difficile une séparation impeccable de l'hystérie avec d'autres névroses et qui obligent à poser l'existence des “névroses mixtes"» ([2], p. 17) ${ }^{2}$.

\footnotetext{
${ }^{1}$ Villa F., chapitre 8 de la deuxième partie : La méthode pour parvenir à une vue d'ensemble des névroses, In : La théorie sexuelle de l'acte. Préliminaires d'une théorie de l'action psychothérapique, ([1], p. 161-173).

${ }^{2}$ Freud S., Les névropsychoses de défense (1894), trad. J. Altounian et A. Bourguignon, In : Euvres Complètes. Psychanalyse, vol. III ([2], p. 17).
} 
Pour saisir l'importance stratégique de cette catégorie clinique dans la démarche freudienne, il faut resituer le statut des névroses ${ }^{3}$ dans le savoir médical au moment où, revenant de Paris, Freud commence, en 1887, sa pratique privée de neurologue. Elles sont le témoignage d'une incompréhension théorique et constituent, au sein des maladies nerveuses, une catégorie nosographique fourre-tout où sont rangées toutes les affections dont la cause organique n'est pas démontrée. Tant pour l'école anatomopathologique que pour l'approche neuropathologique, les névroses sont une «zone d'attente ». La première, d'orientation organiciste centrée sur l'anatomie cérébrale, affirme que, tôt ou tard, sera trouvée la lésion anatomique responsable des névroses. Elle se cantonne logiquement dans la recherche en anatomie cérébrale et elle abandonne, prise de nihilisme thérapeutique, la démarche thérapeutique. L'approche neuropathologique, représentée par l'école de Charcot, était en rupture avec cette tradition organiciste et elle pensait que «l'œuvre de l'anatomie était, somme toute, achevée et la connaissance des maladies organiques du système nerveux, pour ainsi dire, complète » et qu' " il faudrait maintenant s'occuper des névroses » ([4], p. 304).

Parmi ces troubles, sans fondement anatomique, la neurasthénie et l'hystérie sont distinguées plutôt mal que bien, car, leurs tableaux cliniques s'interpénétrant assez souvent, plusieurs auteurs refusent de les différencier. Charcot donnera, du point de vue de la neurologie, ses lettres de noblesse à l'étude de l'hystérie en la différenciant nettement, d'une part, des maladies organiques (paralysies) et d'autre part, de la neurasthénie. Bien que restant attaché à l'approche neuroanatomique, Charcot, à l'opposé d'un Meynert, ne s'en remettra jamais à l'attente d'une nouvelle découverte anatomique. C'est en suivant la voie du diagnostic différentiel ${ }^{4}$ des troubles hystériques et somatiques que Charcot « eut le courage de reconnaitre que la plupart [des] symptômes [hystériques] étaient réels, sans pour autant se départir de la prudence que nécessitait l'insincérité des malades » et qu'il put "édifier une sorte de théorie de la symptomatologie hystérique » ([4], p. 304).

En raison de sa profonde connaissance des maladies organiques du système nerveux, Freud sera réceptif à la façon dont Charcot parvient cliniquement à différencier les névroses des maladies lésionnelles, l'hystérie de la neurasthénie. Il est attentif aux efforts faits pour : « tirer l'hystérie du chaos des névroses » et pour «lui fournir une symptomatologie qui, si multiforme qu'elle soit, ne permet plus d'en mettre en doute les lois et le classement» [4].

\section{Névrose mixte, une voie pour poursuivre la clarification nosographique}

Dans sa pratique privée, Freud reçoit des patients atteints soit d'affections organiques, soit de névroses parmi lesquels prédomine la nervosité commune (autre nom de la neurasthénie) qui le confrontent «à la tâche parfois bien difficile» de «distinguer dès le début entre les affections organiques et neurasthéniques ». Pour y parvenir, il pratique, comme l'indique la lettre à Fließ du 24 novembre 1887, le diagnostic différentiel :

\footnotetext{
${ }^{3}$ Le concept de névrose, introduit par William Cullen en 1777, a connu une indiscutable fortune. Représentant une notion importante dans le système nosologique psychiatrique, il a été rapidement emprunté tant par la langue littéraire que par l'usage courant. Voici la définition donnée, à l'époque, par le dictionnaire médical de Littré et Robin : « nom générique des maladies qu'on suppose avoir leur siège dans le système nerveux et qui consistent en un trouble fonctionnel sans lésion sensible dans la structure des parties ni agent matériel apte à le produire» ([3], p. 29).

${ }^{4}$ Cette méthode est exposée à différentes reprises dans ses Leçons [5] et dans des articles [6-8].
} 
« je m'en suis toujours tenu à un signe distinctif : dans la neurasthénie l'altération hypocondriaque, la psychose d'angoisse, ne doit pas être absente; qu'elle soit niée ou admise, elle se trahit par l'excès de sensations qui se renouvellent, c'est-à-dire par des paresthésies » ([9], p. 31).

Pour poursuivre la clarification nosographique du champ des névroses initiée par Charcot, Freud devra s'opposer non seulement aux théories anatomiques localisatrices de l'hystérie et à celles de la famille névropathique, mais aussi à ces «médecins qui renâclent à toute séparation dans le domaine confus des phénomènes névrotiques, à toute mise en relief d'unités cliniques, d'individus pathographiques » ([10], p. 489) et qui ne reconnaissent pas la nécessité d'un tel travail. Que Breuer ait expérimenté qu'il est possible de guérir par traitement psychique les névroses constitue pour Freud un argument majeur contre l'explication étiologique héréditaire ${ }^{5}$ . Sur l'hérédité, qui relève de l'irréversibilité, aucune action psychique n'est envisageable. Le possible traitement psychique de l'hystérie plaide pour l'étiologie accidentelle et laisse entrevoir qu'il est possible, par le chemin de la régression, d'ouvrir la voie à de possibles remaniements psychiques ${ }^{6}$. C'est sa propre expérience clinique qui conduira Freud à défendre, contre la théorie héréditaire, une théorie de la névrose acquise à la suite de certains accidents de la vie.

C'est dès 1888, que Freud entrevoit que reconnaître le tableau clinique présenté par le patient comme une névrose mixte est une piste pour dépasser la difficulté à former un diagnostic :

«L'hystérie peut se combiner avec maintes autres maladies nerveuses névrotiques et organiques, cas qui rendent alors l'analyse très difficile. La combinaison la plus fréquente est celle avec la neurasthénie, soit que des personnes dont la disposition est quasiment épuisée deviennent neurasthéniques, soit qu'à la suite de circonstances minantes, les deux névroses soient provoquées simultanément. Malheureusement, la plupart des médecins n'ont pas encore appris à distinguer les deux névroses. [...] En outre, l'hystérie locale peut s'associer à des affections locales d'organes isolés. [...] Dans ces cas, la maladie organique devient la cause occasionnelle de la névrose. [...] Par ailleurs, le développement de troubles hystériques exige souvent une sorte de temps d'incubation ou mieux, de latence, pendant lequel la cause continue d'agir dans l'inconscient. C'est ainsi qu'une paralysie hystérique ne surgit presque jamais immédiatement après un traumatisme » ([12], p. 164).

Les éléments avancés dans ce passage apparaissent d'autant plus importants qu'ils sont l'introduction de la temporalité comme élément central pour penser la genèse des névroses et la spécificité de l'événement psychique. Le temps d'incubation et la proposition de considérer que l'état manifeste présent doit être déconstruit pour en reconstruire les différents temps de sa constitution préfigurent l'idée de l'après-coup et de ses effets psychiques.

\footnotetext{
${ }^{5}$ Si Freud n'accorde pas à l'hérédité le rôle principal étiologique, cela ne veut pour autant pas dire qu'il retire toute importance à la prédisposition, mais celle-ci n'a plus la signification que lui donnait la théorie héréditaire ([11], p. 95-96).

${ }^{6}$ «Car que prétend faire thérapeutiquement avec l’hérédité pour étiologie ? Elle a depuis toujours existé chez le malade et continuera d'exister jusqu'à la fin de celui-ci. Elle n'est, en soi et pour soi, propre à faire comprendre ni la survenue épisodique d'une névrose ni sa cessation par traitement. Elle n'est rien d'autre qu'une condition de la névrose, certes une condition d'une indicible importance, mais, cependant, une condition surestimée au détriment de la thérapie et de la compréhension théorique ». Freud S. Sur la critique de la «névrose d'angoisse » (1895). In : Euvres Complètes. Psychanalyse, vol. III, ([2], p. 77).
} 


\section{Traitement comme méthode de diagnostic}

L'application de « la méthode de Breuer de traitement du symptôme hystérique par exploration et par abréagir sous hypnose » ([11], p. 206-207) ${ }^{7}$ exigera de surmonter deux difficultés dont le dépassement ouvrira la voie à l'élaboration de la méthode et de la théorie psychanalytiques. La première est bien connue : « toutes les personnes qui montrent des symptômes indubitablement hystériques ne sont pas hypnotisables ». La seconde, qui a moins retenu l'attention, nous semble pourtant cruciale, elle est déterminée par la conception des névroses mixtes. Freud remarque que la méthode cathartique s'adressant à l'hystérie, une fois écartée la cause organique, il fallait, pour l'appliquer, « prendre position sur la question de ce qui caractérisait essentiellement l'hystérie et par quoi celle-ci se différenciait des autres névroses ». Et, en raison même du fait qu'il n'existe probablement pas de névrose pure, mais seulement des tableaux de névrose mixte, il est impossible à partir des seuls symptômes de procéder au diagnostic. Il faut avoir reconstruit le mécanisme psychique qui prédomine dans la formation du symptôme pour pouvoir nommer la névrose. Surgit alors la difficulté suivante : « Il est fort malaisé de se faire une opinion exacte d'un cas de névrose avant d'avoir soumis celui-ci à une analyse approfondie, qui ne peut être différente de celle utilisée par Breuer. La décision du diagnostic et du genre de thérapie doit pourtant être prise avant une telle connaissance approfondie ». C'est en surmontant «tous les doutes » liés à cette deuxième difficulté que surgira «le plan» de traiter toutes les névroses de la même façon que l'hystérie. Cela permit le constat que :

« parfois, alors, les résultats thérapeutiques, en dépit du diagnostic d'hystérie, restaient bien médiocres et l'analyse elle-même ne mettait rien d'important en lumière. D'autres fois, j'essayais de traiter par la méthode de Breuer des névrosés que nul certainement n'aurait tenus pour hystériques et sur lesquels néanmoins le procédé agissait et parfois même supprimait les symptômes. C'est ce qui [lui] arriva, par exemple dans des cas d'idées obsédantes véritables, du type de Westphal, cas qui ne rappelaient l'hystérie par aucun trait» [11].

Après avoir contribué à défaire l'étiologie héréditaire, l'application de la méthode cathartique, en montrant que «le mécanisme révélé dans la "Communication préliminaire" ne pouvait être regardé comme un signe pathognomonique de l'hystérique », faisait apparaître que l'on pouvait entreprendre aussi avec les autres névroses la recherche «de l'étiologie et le genre du mécanisme psychique ». L'application du traitement cathartique devenait de ce fait un instrument de diagnostic.

Le résultat d'une telle démarche sera de trois ordres :

- premièrement, la reconnaissance de l'existence de névroses acquises entraîne la recherche des causes étiologiques et permet de découvrir que les facteurs étiologiques sont de nature sexuelle ;

- deuxièmement, il apparaît que différents facteurs sexuels produisent différents tableaux cliniques de névrose ;

- troisièmement, il s'avère que les variations étiologiques peuvent servir à différencier les névroses et à établir une stricte délimitation nosographique.

\footnotetext{
${ }^{7}$ Freud S. Zur Psychotherapie der Hysterie (1895) in Studienausgabe, Schriften zur Behandlungstechnik, Ergänzungsband, ([13], p. 50-52).
} 
C'est ainsi qu'à partir de la neurasthénie sont dégagées la névrose obsessionnelle, l'hystérie d'angoisse qui sont rangées au côté de l'hystérie dans la nouvelle entité des psychonévroses et, dans le même temps, par la distinction de la névrose d'angoisse de la neurasthénie, est constituée la classe des névroses actuelles.

\section{Névroses mixtes : une leçon de méthode}

Du point de vue de sa clientèle, l'utilisation du traitement cathartique eut un effet sélectif, il éloigna du cabinet de Freud ces malades nerveux organiques dont il était pourtant un expert du point de vue du diagnostic. Et, la pratique lui confirma que le tableau clinique le plus fréquemment rencontré était celui de la névrose mixte : il était quasi impossible de trouver une forme névrotique à l'état pur. Dans ce contexte, il devenait clair pour Freud que Krafft-Ebing avait raison d'affirmer que la psychiatrie de son époque méritait tout au plus le nom de science descriptive et qu'il lui fallait encore faire d'immenses efforts avant de s'élever au rang de science explicative [14], ([15], p. 123). Admettre l'idée des névroses mixtes fut le préalable qui, guidant l'observation clinique, permit d'introduire un principe d'ordre dans ce fouillis hétéroclite de tableaux cliniques que l'on désignait, faute de mieux, comme neurasthénie ${ }^{8}$. Pour Freud, c'est la sous-estimation par ses contemporains de la capacité des névroses de constituer un "tel mélange clinique » qui rendait difficile leur distinction. Pour pouvoir avancer tant du point de vue du diagnostic que du point de vue théorique, il fallait aller au-delà du tableau clinique manifeste et reconnaître ce dernier comme produit résultant de la condensation de plusieurs autres tableaux. C'est cette condensation qui s'avère porteuse de confusion et qui permet de confondre entre elles les différentes formes de névrose en les réduisant à une seule forme générique : la neurasthénie.

La position de Freud est clairement exposée, entre autres, dans la réponse qu'il fait à KrafftEbing lors de la discussion de sa conférence sur le «Mécanisme des représentations de contrainte et des phobies $»$, mais on la retrouve dans bien d'autres textes $[11]^{9}$.

«La question de savoir si on doit ranger les représentations de contrainte dans la neurasthénie ou comme il le pense, les expliquer comme une névrose sui generis, est avant tout, une question de nomenclature, donc de convention, d'opportunité. La réponse à cette question dépend aussi du point de vue que l'observateur soutient en général en ce qui concerne les névroses. Pour qui met le poids principal sur l'occurrence simultanée, l'occasion ne se trouvera guère de séparer les représentations de contrainte de la neurasthénie. Mais, si quelqu'un comme lui (Freud) tient compte en premier lieu de l'étiologie et du mécanisme des névroses et professe la conception que les névroses le plus souvent observées sont "mixtes", pour celui-là neurasthénie, névrose d'angoisse, représentations (névroses) de

\footnotetext{
${ }^{8} \mathrm{La}$ neurasthénie a eu son heure de gloire. C'est un terme générique sous lequel on rangeait toutes les affections dont on ne savait quoi faire du point de vue anatomopathologique. Beard l'avait présentée dans deux articles (1882 et 1884) comme un syndrome d'allure dépressif, chronique, avec prédominance de troubles diffus corporels, se traduisant par des somatisations avec désordres fonctionnels et plus particulièrement avec des douleurs. Cette névrose était le fruit de la civilisation moderne et elle apparaissait «à la suite d'égarements sexuels (surtout de la masturbation) », d'autres auteurs mettaient en relief le rôle du coït interrompu.

${ }^{9}$ Freud S. Du bien-fondé à séparer de la neurasthénie un complexe de symptômes déterminé, en tant que « névrose d'angoisse » [1894 (1895b)], In : Euvres Complètes. Psychanalyse, vol. III [2], Freud S. L'hérédité et l'étiologie des névroses (1896). Texte original en français, In : Euvres Complètes. Psychanalyse, vol. III [2].
} 
contrainte se départagent indubitablement. Ceci est donc un point qui ne saurait être traité en dehors d'un plus vaste contexte ${ }^{10}$.

Faire l'hypothèse de l'existence des névroses mixtes permet donc de dépasser le leurre de «l'occurrence simultanée » des signes et des symptômes qui n'est qu'un effet d'optique produit par la combinaison de différentes névroses entre elles. Dans le tableau clinique actuel, apparaissent divers symptômes que l'on a tendance, en raison de leur contiguïté temporelle manifeste, à considérer trop rapidement comme étant étroitement reliés entre eux ; ils appartiendraient à la même affection, ils relèveraient de la même cause. Or, cela Freud va le remettre en question. La première tâche qui s'impose est de parvenir à extraire du tableau les complexes de symptômes qui correspondent à la même affection et qui résultent d'un même mécanisme de production. Son point de départ est la séparation établie entre neurasthénie et hystérie. Deux observations cliniques ont pu être faites : d'une part, des symptômes qui se présentent simultanément n'appartiennent pas obligatoirement à la même névrose : certains relèvent de la neurasthénie, d'autres de l'hystérie. D'autre part, même quand un symptôme appartient à l'une et à l'autre névrose, cela ne signifie ni qu'il a la même origine, ni la même fonction. En effet, un symptôme apparu, dans le contexte de la formation d'une névrose donnée, une fois constitué, peut devenir, au moment de la formation d'une autre névrose, un des matériaux qu'elle utilisera pour s'exprimer. Ainsi, par exemple, Freud notera à propos du cas de Katarina qu'il

« est le modèle de ce que j'ai appelé la peur virginale, c'est une combinaison de névrose d'angoisse et d'hystérie, la première crée les symptômes, la seconde les répète et travaille avec eux » ([11], p. 209).

En ne retenant que la simultanéité de présentation, on ne pouvait que tout confondre et on se condamnait à ne rien pouvoir distinguer. Dès lors qu'est reconnu que la contiguïté ne fait pas nécessairement lien et que le symptôme n'est pas naturellement univoque, il faut donc défaire en pensée ce qui présentement se donne comme une unité et il est nécessaire, préalablement à tout diagnostic, de déterminer quels sont les symptômes qui sont connectés entre eux par un même mécanisme psychique : celui qui les a produits. La décomposition du tableau en fragments met alors en lumière que tous les symptômes actuellement présents n'appartiennent pas nécessairement au tableau symptomatique de la même affection.

L'expérience clinique nous apprend deux leçons. La première nous oblige à envisager qu'un symptôme, qui apparaît dans deux cas différents et dont la ressemblance manifeste de forme inciterait à dire que c'est le même symptôme, ne l'est peut-être qu'en apparence et non pas en vérité. Ce n'est que la découverte du mécanisme de sa production qui rend possible la compréhension du symptôme et il peut, en effet, s'avérer que derrière la même forme symptomatique, œuvrent des mécanismes différents. Reconnaître qu'il est possible, à partir du mécanisme qui les a engendrés, d'apparenter nosographiquement entre eux certains symptômes, constitue une deuxième leçon clinique. Elle conduit à penser que chacun des symptômes de cet ensemble peut en venir, sous certaines circonstances, à représenter, à lui seul, le tout de la maladie : les symptômes sont, en effet, entre eux dans un rapport de possible substitution de l'un par l'autre. Dans certains cas, la maladie se manifestera par la présence dans le tableau

\footnotetext{
${ }^{10}$ Freud S. Compte rendu par Freud de sa conférence sur le « Mécanisme des représentations de contrainte et des phobies » [1895(1895h)], trad. P. Cotet, In : Euvres Complètes. Psychanalyse, ([2], p. 89).
} 
pathologique de l'ensemble des symptômes et, dans d'autres, elle ne se révèlera discrètement que par la présence d'un unique symptôme (qui représentera pourtant l'ensemble de la maladie).

Dans cette démarche, Freud sera amené à différencier les conditions étiologiques des facteurs étiologiques. Les conditions sont multivoques : elles peuvent produire plusieurs névroses, les facteurs sont univoques et sont spécifiques à chaque affection. Les conditions changent, les facteurs sont uniques et intimement corrélés à l'affection. Les facteurs étiologiques ne peuvent qu'en être abstraits, les conditions n'ont pas à être devinées, elles sont le plus souvent connues, ce qui est inconnu c'est leur signification psychique effective pour le sujet. Le rapport entre les différents facteurs étiologiques, présents dans le tableau de la névrose mixte, peut être soit un simple effet du hasard, soit au contraire que l'un d'entre eux a entraîné l'effet de l'autre.

Notons que, dès lors que l'attention se porte sur le mécanisme, surgit la question de la fonction, de la visée, de la finalité et se produit immanquablement un changement de paradigme dans la recherche étiologique qui acquiert là de nouveaux fondements. L'actualité du symptôme ne tient plus au temps de sa manifestation, mais à ce qui est rendu actuel par ce qu'il accomplit, par ce qu'il réalise de la visée à laquelle a répondu sa formation. Dès lors, comme le pose Freud, dans l'analyse des «névroses mixtes», il faut non seulement partir de l'idée que quelque chose a donné lieu au symptôme qui est une réaction à ce quelque chose: il y a eu causation du symptôme ([11], p. 207) mais que, de plus, « là où se trouve une névrose mixte, on peut mettre en évidence un mélange de plusieurs étiologies spécifiques $»^{11}$.

Comme nous le voyons, la notion de névrose mixte constitue une leçon de méthode quant à la genèse du tableau clinique. À nous en tenir à la forme actuelle manifeste, nous méconnaîtrions le travail du temps, travail non d'un temps linéaire, mais d'une temporalité singulière qui est celle de l'après-coup, travail donc de la régression et de la répétition. Cette notion correspond à l'idée freudienne que toute forme psychique n'est qu'une forme asymptotique. Toute forme n'est que tendance vers une forme véritablement adéquate, mais inatteignable. Elle n'est pas un aboutissement mais un en chemin vers : une motion vise à s'accomplir et n'y parvient que de manière approximative, inachevée. Forme qui, n'accomplissant pas en acte ce qui était en puissance, se voit soumise au travail de ce qui, restant en puissance, contraint à sortir de la forme déjà constituée, obligeant à supporter la déformation imposée par les accidents de la vie qui sont autant de renforcements de la contrainte à accomplir la motion.

\section{Mode de pensée psychanalytique et symptôme}

La méthode freudienne ouvre, face au fait symptomatique, une autre voie que celle de la description d'un tableau des symptômes dont l'exhaustivité ne parvient pas à masquer la stérilité tant du point de vue de l'efficacité thérapeutique que de l'élucidation des mécanismes psychiques présidant à sa formation. Pour l'approche psychanalytique, dresser le tableau clinique n'est que le temps préliminaire à partir duquel il sera procédé, par analyse, à la décomposition de celui-ci en fragments. La reconnaissance de la «significativité » de ces fragments implique de les situer non seulement dans le temps historique auquel ils appartiennent, mais également

\footnotetext{
${ }^{11}$ Freud S. Du bien-fondé à séparer de la neurasthénie un complexe de symptômes déterminé, en tant que « névrose d'angoisse » [1894 (1895b)], In : Euvres Complètes. Psychanalyse, vol. III ([2], p. 55).
} 
de repérer les remaniements qu'ils ont subis au cours d'un temps qui ne respecte pas l'ordre chronologique linéaire et qui est régi par la logique temporelle de l'après-coup. Celle-ci n'est rendue possible qu'en raison du fait qu'au niveau inconscient, les traces mnésiques les plus anciennes comme les plus récentes ont la même actualité.

La méthode psychanalytique opère le passage d'un tableau où l'accent était mis sur le manifeste que constitue l'occurrence simultanée des traits symptomatiques à une présentation métapsychologique de l'affection psychique. Ce qui caractérise cette présentation est de permettre la construction par inférence des mécanismes psychiques qui constituent le fondement de la «maladie». Il ne s'agit plus de décrire, mais de réinscrire la maladie dans le temps d'une vie [16,17], dans l'histoire des destins « affectifs » de ce qui arriva sans trouver lieu psychique d'inscription. Dans ce passage de l' «objectivité »d'un tableau pathologique à la mise en évidence des "motifs », se découvre la subjectivité qui ouvre dans l'événementialité psychique.

La rupture qu'opère le mode de penser psychanalytique avec la méthode descriptive a pour conséquence un changement radical dans la perception du symptôme. Ce dernier n'est plus objectivé, il n'est plus un objet dont l'évidence serait indiscutable du point de vue d'une perception objective. Il cesse d'être un trait distinctif par lui-même pour devenir un élément inscrit topiquement dans une dynamique où il est associé à d'autres éléments avec lesquels il constitue un complexe. Ce dernier caractérise une organisation psychique qui est déterminée par des motifs psychiques et qui vise à atteindre certains buts. Ceux-ci sont accomplis partiellement et de manière déformée, déguisée dans le symptôme. Le symptôme n'est donc plus un phénomène complètement stabilisé, de nature statique (et cela bien qu'il soit aussi le lieu d'une certaine stase), mais un point critique. Point critique est à entendre de manière polysémique. Il est le point où se représente une crise passée qui reste actuelle dans le symptôme qui en constitue le "symbole mnésique ». Le symptôme est à la fois l'expression de ce qui fut jadis inventé comme issue de la crise et le lieu où agit encore manifestement et douloureusement ce dont le sort ne fut assuré ni dans la décharge, ni psychiquement. La création de symptôme répond à la nécessité que survienne un changement d'état. Il vise à sortir d'un état de conflictualité exacerbée (où l'excitation pulsionnelle a dépassé le seuil du supportable) et à revenir à un état idéal d'inertie ou pour le moins de constance ([9], p. 604-605).

Au niveau de la visée qui préside à son invention, le symptôme se construit, dans la crise et par rapport à elle, comme une issue qui se voudrait définitive et il tend à s'instaurer comme un état permanent stable que plus rien ne remettrait en cause. Or, en pratique, il n'en est pas ainsi - ou seulement en partie. En réalité, le symptôme est le lieu dynamique d'une activité pulsionnelle et le résultat d'une modification autoplastique et il est, de plus, de manière déguisée, une activité exercée sur certains objets du monde. Ceux-ci, en devenant les supports du transfert, subissent le traitement psychique que leur impose le fait d'être inclus, à l'insu de l'individu et de l'objet, dans les formations symptomatiques du sujet. Dans le traitement psychanalytique, il devient impossible de penser et de saisir le symptôme en lui-même, sa fonction ne peut se découvrir qu'en devinant l'activité autoplastique et la modification alloplastique qu'il accomplit dans le transfert.

\section{Le symptôme ne prend sens que dans le transfert : savoir par la souffrance}

L'analyse des symptômes ne se fait pas directement, frontalement, elle ne s'effectue que de manière indirecte en saisissant leur fonction et leur raison à partir de l'analyse de leurs effets 
transférentiels sur le psychanalyste. C'est dans le transfert, par lui, que les symptômes retrouvent leur valeur psychique et ce n'est qu'à partir de la névrose de transfert qu'ils peuvent être entendus. Mais, faut-il rappeler qu'avant d'analyser le transfert, il faut, comme l'écrivait Freud au pasteur Pfister, avoir supporté de porter la croix qu'il représente pour le psychanalyste.

Nous ne devons pas sous-estimer, cela a son importance du point de vue de la recherche psychanalytique, que c'est un savoir par la souffrance que rend possible le mode de penser psychanalytique. Nous nous inscrivons à la suite du travail de P. Fédida sur le pathéi mathos (savoir par la souffrance) ${ }^{12}$. Ce travail s'ouvrait sur une citation de l'Agamemnon d'Eschyle dont je ne résiste pas à faire la citation :

«Celui qui a ouvert aux mortels le penser posant qu'ils tiendraient principalement leur savoir par la souffrance. Dans le sommeil, le mal où revit la peine coule devant le cœur. Et la pensée sûre est venue à ceux qui n'en voulaient pas ${ }^{13}$.

Il nous semble qu'il en va ainsi dans le mode de penser psychanalytique : la pensée sûre vient à ceux qui n'en voulaient pas ${ }^{14}$. À propos du pathéi mathos, Fédida soutenait qu'on ne saisissait le psychopathologique en son fondement tragique (au-delà des dramatisations psychologiques œdipiennes) qu'à tenir ensemble ces deux mots et à penser à partir de la dissymétrie qu'ils ouvrent, en nous, entre l'expérience vécue et le savoir que nous en avons, entre ce qui est arrivé et ce qui a eu lieu psychiquement.

Dans la progression de notre pensée, s'est opéré un déplacement d'importance : ce qu'il faut livrer au travail de décomposition, ce n'est plus le tableau des symptômes que présente le patient, mais le tableau que constitue la scène transférentielle qui s'est construite dans la situation psychanalytique. Pour clore, nous avancerons une piste de recherche future. La névrose de transfert n'équivaut-elle pas à la formation d'une névrose mixte dont la décomposition analytique permet de reconstruire le destin des pulsions qui s'est accompli depuis la névrose infantile jusqu'à la névrose clinique qui a conduit le patient au traitement ? Si une telle hypothèse s'avérait pertinente, la névrose mixte serait un équivalent dans notre pratique de la pierre de Rosette.

\section{Références}

[1] Villa F. La théorie sexuelle de l'acte. Préliminaires d'une théorie de l'action psychothérapique [Thèse de Doctorat de Psychopathologie Fondamentale et Psychanalyse]. Paris : Université Paris-VII-Denis-Diderot; 2000.

[2] Freud S. CEuvres Complètes. Psychanalyse, vol. III. Paris: PUF; 1989.

[3] Andersson O. Studies in the prehistory of psychoanalysis: the etiology of psychoneuroses and some related themes in Sigmund Freud's scientific writings and letters 1886-1896. Stockholm: Svenska Borkförlaget Norstedts; 1962.

\footnotetext{
${ }^{12}$ Fédida P. Tradition tragique du psychopathologique. À propos du pathei mathos de l'Agamemnon. In : Crise et contre-transfert, ([18] p. 19-36).

${ }^{13}$ Eschyle. Agamemnon, vers 176-181. In: Bollack J., Judet de La combe P., editors. L' "Agamemnon" d'Eschyle, [19].

${ }^{14}$ Cela n'est pas sans évoquer ce que Bion W.R. écrit : « quiconque s'apprête à voir un patient demain doit, à un moment quelconque, éprouver de la peur. Il devrait se trouver dans tout cabinet de consultation deux individus assez effrayés : le patient et le psychanalyste. S'ils ne le sont pas, pourquoi se soucient-ils de découvrir ce que tout le monde sait sans vouloir le savoir?», ([20], p. 6).
} 
[4] Freud S. Rapport sur mes études à Paris et à Berlin poursuivies grâce à une bourse de voyage accordée par le fonds du jubilé de l'université (1886). Revue Française de Psychanalyse 1956;20(3):299-306 [trad. A. Berman].

[5] Charcot JM. Leçons sur les maladies du système nerveux. Euvres complètes, T. III. Paris: Delahaye et Lecrosnier; 1887.

[6] Charcot JM. Contribution au diagnostic différentiel entre l'hystérie et les maladies organiques du cerveau. Arch Neurol 1892;24:387-422.

[7] Charcot JM. Contribution au diagnostic différentiel entre l'hystérie et les maladies organiques du cerveau. Arch Neurol 1893;25:41-64.

[8] Charcot JM. Sclérose latérale amyotrophique ou amyotrophie hystérique ? Difficultés de diagnostic. Arch Neurol 1893;25:161-78.

[9] Freud S. Lettres à Wilhelm Fließ, 1887-1904. Paris: PUF; 2006 [trad. Kahn F, Robert F.].

[10] Freud S. Conférences d'introduction à la psychanalyse (1917). Paris: Gallimard; 1999 [trad. F. Cambon].

[11] Freud S, Breuer J. Études sur l'hystérie (1895). Paris: PUF; 1956 [trad. A. Berman].

[12] Freud S. Hystérie (1888). Cah Confront 1982;7:153-70 [trad. M. Borch-Jacobsen, Ph. Kœppel, F. Scherrer].

[13] Freud S. Studienausgabe, Schriften zur Behandlungstechnik, Ergänzungsband. Frankfurt am Main: S. Fischer; 1989.

[14] Krafft-Ebing R. Lehrbuch der Psychiatrie auf klinischer Grundlage für pracrtische Ärzte und Studirende, T. 1. Stuttgart: Enke; 1879.

[15] Hirschmüller A. Joseph Breuer. Paris: PUF; 1991 [trad. M. Weber].

[16] Hippocrate. De l'art médical. Paris: Livre de poche; 1994 [trad. Littré E.].

[17] Villa F. L'exigence d'une éthique du bien dire. À propos du dialogue du médecin avec ses patients. In: Brun D, editor. Sixième Colloque de Médecine et Psychanalyse. La fabrique des bébés : passions pour l'embryon. Paris: Ed. Études freudiennes; 2004. p. 89-99.

[18] Fédida P. Crise et contre-transfert. Paris: PUF; 1992 (p. 19-36).

[19] Bollack J, Judet de La combe P, editors. L' «Agamemnon » d'Eschyle. Presses Universitaires de Lille et Éditions de la Maison des Sciences de l'Homme; 1981-1982.

[20] Bion WR. Entretiens psychanalytiques. Paris: Gallimard; 1980 [trad. B. Bost]. 